\section{Zur Pharmakologie des Tannins und seiner Anwendungsformen.}

\section{Von L. Lewin in Berlin.}

Experimentelle Ergebnisse führten mich vor mehr als zwei Dezennien dazu, einmal die alte, rein empirische Anwendung der gerbsäurehaltigen Mittel für örtliche und entferntere Einwirkungen wissenschaftlich als zutreffend zu erweisen, sodann auch neue Anwendungsformen auf Grund meiner Forschungen aufzustellen. Ich konnte an Tieren dartun, daß ein Teil der Gerbsänre als solcher durch die Nieren ausgeschieden wurde. ${ }^{1}$ ) Dieses Ergebnis ist später von Stockmann ${ }^{2}$ ) bestätigt worden.

Einwendungen, die gegen dasselbe geltend gemacht wurden, ${ }^{3}$ ) hatte ich keinen Grund noch einmal zu widerlegen, da ich meine Untersuchungen für zuverlässig hielt und mir deswegen die Zeit zu Wiederholungen solcher Versuche velloren schien. Stock$\operatorname{mann}^{4}$ ) hat sich dieser Mühe unterzogen und von neuem erwiesen, dal durch die von mir angewendete Methode des Tanninnachweises es gelingt, bei Kaninchen Gerbsäure im Harn nachzuweisen und daß, wenn man Alkalitannat einführt, auch bei Hunden und Menschen stets Gerbsäure in den Harnen zu finden war.

Es kann als zweifellos angesehen werden, daf individuelle Verhältnisse, wie z. B. die größere oder geringere lebhaftigkeit der Umsetzungen im Körper, bestimmend auf das Schicksal der Gerbsäure cinwirken können, sodaß bald nur sehr wenig. bald mehr derselben neben Gallussäure zur Ausscheidung kommt. Innmerhin ist die Möglichkeit der Ausscheidung unveränderter Gerbsäure durch die Nieren und damit die Möglichkeit einer Fernwirkung erwiesen.

Die Art des Eintretens der Gerbsäure in die Blutbahn von den gewöhnlichen Resorptionsorten aus, erfolgt nach meinen Versuchen durch Ueberführung in Alkalitannat, dem ebenfalls noch starke adstringierende Wirkungen zukommen. Diese Erkenntnis mußte notwendig dazu führen, das Alkalitannat, dem eiweißfällende Eigenschaften nicht mehr zukommen, ${ }^{5}$ ) für die Therapie zu empfehlen. Von einer solchen, mit Natriumbikarbonat neutralisierten Lösung von Gerbsäure wurde und wird reichlich Gebrauch gemacht. Ribbert6 konnte bei künstlich albuminurisch gemachten Tieren nach intravenöser Beibringung einer solchen Lösung ein Fehlen des Eiweiß in den meisten Glomerulis und in den übrigen ein nur geringes Vorhandensein feststellen.

Das chemische Verhalten der Gerbsäure im Magen nötigte mich aber, noch eine andere Anwendungsmethode zu empfehlen. Die lebhafte Beziehung, die das Tannin zu Eiweib, also auch zu dem Gewebseiweiß besitzt und die sich in der Bildung von Tanninalbuminat äußert, legten es nahe, um Reizungen der Magenschleimhaut zu vermeiden, die Einführung von Tanninalbuminat zu empfehlen, für dessen Verschreibung ich wiederholt Vorschriften gegeben habe. ${ }^{7}$ ) Denn die örtliche gerbende Wirkung beruht, wie ich zuerst darlegte, ${ }^{8}$ ) darauf, daß die intrazellulären Flüssigkeiten und der gelöste Zellsaft chemisch verändert werden und dadurch auch die Morphologie des Gewebes leiden müsse.

Durch Verabreichung von in Wasser verteiltem Tanninalbu. minat müßten sich diese Nachteile vermeiden lassen. Es sind tatsächlich damit auch gute Resultate klinisch erzielt worden.

Nun hat Gottlieb 16 Jahre später, ohne meiner Ergebnisse

1) Lew in , Archiv für pathologische Anatomie 1880, Bd. 81. Deutsche medizinisch Wochenschrift 1881, No. 15. - 2) St ock m a n n, British medical Journal 1886, Bd. 2, S. 1077 - 3) Rost und Spickenboom, Archiv für experimentelle Pathologle 1897, Bd. 38. 4) Stockmann, Archiv für experimentelle Pathologie 1898, Bd. 40, S. 147. - - 5) Di g) Senteilige Angabe (Geb hardt, Archiv für klinische Medizin Bd.66) 147.5 - 5) Die gegenteilige Angabe (Gebhard, Archiv für klinische Medizin $\mathrm{Bd}$. 66) beruht, wie aus die medizinische Wissen, auft 1889 , S. 36 . 7) Lew in. Deutsche mo schrift 1881 , No. 15 , und 1882 , No. 6 :

$$
\begin{aligned}
& \text { Sol. acid. tannic. } 2-5: 150,0 \\
& \text { Adde agitando }
\end{aligned}
$$$$
\text { Album. solut. ovi un }
$$

8) Archiv für pathologische Anatomie 1. c. S. 15 zu gedenken, ein Tanninalbuminat angegeben, das patentiert worden ist und unter dem Namen Tannalbin verkauft wird.1) Meine experimentellen Ergebnisse sind hier fabrikatorisch mit der Modifikation nutzbar gemacht worden, daß das Tanninalbuminat trocken bei hoher Temperatur eine gewisse Zeit lang erhitzt wurde, wodurch es der Magenresorption entzogen werden, aber im Darm noch zerlegbar bleiben soll. Nach Gottlieb gelangt das ganze in dem Präparat enthaltene Tannin bis in den Darm und ,dürfte bei der Schwerlöslichkeit des Präparates wohl auch die untersten Darmabschnitte erreichen“. Beweise hierfür sind nicht erbracht worden. Dagegen ist erwiesen worden, dab nach Eingabe von Tannalbin bei Tieren im Harn Gallussäure erscheint, wie nach Eingabe von Tannin, daß das gleiche Verhalten der Kot zeigt und nur eine Katze einmal mit dem Kote Gerbsäure was aber wahrscheinlicher ist, unverändertes Tannalbin - ausschied.

Ich habe mich auch aus dem klinischen Material nicht überzeugen können, daß diese Form der Tannindarreichung irgend welche Vorteile vor der bisherigen hat. Vor allem bin ich aber davon überzeugt, daß das von mir angegebene Tanninalbuminat dem Tannalbin an Wirkung mindestens gleichkommt, da ich erweisen kann, daß es trotz seiner Löslichkeit im Magensafte sich auch im oberen Darm findet.

Als besonders befremidlich mul ich es aber bezeichnen, dal neuerdings von Zeit zu Zeit sich in Journalen kurze Mitteilungen finden, in denen orakelt wird, dab "das nach patentiertem Verfahren von Prof. Gottlieb therapeutisch verwendbar gemachte Tannineiweiß nicht durch andere Tanninalbuminate arzneilich orsetzt werden kann". Nichts hiervon ist richtig, $d a$ jedes Tannin albuminat das Tannalbin ersetzen kann. Was drolligste ist aber, dab in einer solchen votiz auch darauf hingewiesen wird, „dab ein dem Tannalbin therapeutisch gleichwertiges Präparat ohne Verletzung der Patentrechte nicht dargestellt werden darf". Ferner wird die Belehrung gegeben, dals "dio Verwendung eines minderwertigen Produktes z. B. bei der Anwendung für Säuglinge, eine große Verantwortung einschließen würde".

Demgegenüber weise ich darauf hin, dah das von mir empfohlene Tanninalbuminat von Jedem hergestell t werden darf, in jeder Form verwendbar ist und Nebenwirkungen nicht erzeugt.

Ich möchte hier nochmals geradc auf die chronische Verwendung ${ }^{2}$ ) bei nephritischen Zuständen hinweisen, da hierbei parenchymatöse und Gefäßwirkungen durch Tannin zu stande kommen müssell. Desgleichen wären Versuchc mit chronischer Darreichung dünner Tanninalbuminatlösungen bei Tuberkulösen sehr wünschenswert. 